\title{
Serum Proinflammatory Mediators at Different Periods of Therapy in Patients With Multiple Myeloma
}

\author{
Irfan Kuku, ${ }^{1}$ Mehmet Refik Bayraktar, ${ }^{2}$ Emin Kaya, ${ }^{1}$ Mehmet Ali Erkurt, ${ }^{1}$ \\ Nihayet Bayraktar, ${ }^{3}$ Kerim Cikım, ${ }^{1}$ and Ismet Aydogdu ${ }^{1}$ \\ ${ }^{1}$ Department of Hematology, Medical Faculty, Turgut Ozal Medical Center, Inonu University, 44069 Malatya, Turkey \\ ${ }^{2}$ Department of Medical Microbiology, Medical Faculty, Turgut Ozal Medical Center, Inonu University, 44069 Malatya, Turkey \\ ${ }^{3}$ Department of Clinical Biochemistry, Medical Faculty, Turgut Ozal Medical Center, Inonu University, 44069 Malatya, Turkey
}

Received 22 December 2004; accepted 1 March 2005

\begin{abstract}
Multiple myeloma (MM) is a malignant disease characterized by the clonal proliferation of plasma cells within the bone marrow. Several cytokines have been demonstrated to be involved in the control of growth, progression, and dissemination of MM. We determined serum levels of interleukin- $\beta$ (IL-1 $\beta$ ), soluble interleukin-2 receptor (sIL-2R), interleukin-6 (IL-6), interleukin-8 (IL-8), tumor necrosis factor- $\alpha$ (TNF- $\alpha$ ), and C-reactive protein (CRP) in 14 newly diagnosed MM patients. The median age of the patients was $63.4 \pm 10.8$ years and all of the patients were stage III (classified according to the Durie-Salmon classification). The same parameters were measured in 15 healthy controls. In addition, we also examined the effects of vincristine-adriamycin-dexamethasone (VAD) therapy on the same parameters and mediators as well as the relationship among the parameters in the same patient groups. The serum concentrations of TNF- $\alpha$, IL- $1 \beta$, sIL-2R, IL-6, IL-8, and CRP $(18.6 \pm 3.7 \mathrm{pg} / \mathrm{mL}, 10.1 \pm 2.8 \mathrm{pg} / \mathrm{mL}, 730 \pm 220 \mathrm{U} / \mathrm{mL}$, $11.4 \pm 3.3 \mathrm{pg} / \mathrm{mL}, 23.9 \pm 8.3 \mathrm{pg} / \mathrm{mL}$, and $49.9 \pm 19.5 \mathrm{mg} / \mathrm{dL}$, resp) were significantly higher in newly diagnosed MM patients than in healthy controls $(P<.0001)$. All of the parameters were found to be significantly reduced after chemotherapy. In conclusion, we found that after the VAD therapy, the level of these cytokines which are thought to play an important role in the pathogenesis of $\mathrm{MM}$ was significantly suppressed. This is the first study demonstrating strong impact of VAD treatment on circulating mediators of sIL-2R and IL-8 levels parameters.
\end{abstract}

\section{INTRODUCTION}

Multiple myeloma (MM) is a B-cell malignancy characterized by the accumulation of a clonal population of plasma cells in the bone marrow that secretes a monoclonal immunoglobulin protein. Bone marrow stromal cells and myeloma cells produce several proinflammatory cytokines that play an important role in the pathogenesis of multiple myeloma. Of these, Interleukin-6 (IL6 ) is known as a growth and survival factor in multiple myeloma via activation of extracellular signal-regulated kinase and phosphatidylinositol 3-kinase signaling cascade [1]. Another proinflammatory cytokine such as Interleukin- $1 \beta$ (IL-1 $\beta$ ), a potent osteoclast-activating factor, can increase the expression of adhesion molecules and induce paracrine IL- 6 production $[2,3]$. These biologic effects of IL- $1 \beta$ closely parallel several of the clinical

Correspondence and reprint requests to Irfan Kuku, Department of Hematology, Medical Faculty, Turgut Ozal Medical Center, Inonu University, 44069 Malatya, Turkey; ikuku@inonu.edu.tr features of human myeloma, such as osteolytic bone lesions, homing of myeloma cells to the bone marrow, and IL-6-induced cell growth [3]. Similar to IL-1 $\beta$, tumor necrosis factor- $\alpha$ (TNF- $\alpha$ ) is also a potent bone-resorbing proinflammatory cytokine that may contribute to the development of the osteolytic bone disease observed in patients with MM $[2,4]$. C-reactive protein (CRP) is produced by hepatocytes in response to inflammation and infection and strongly correlated with proinflammatory cytokines particularly IL-6 [5]. On the other hand, soluble interleukin-2 receptor (sIL-2R) can increase during some inflammatory processes and malignant disorders $[6,7]$. Levels of serum sIL-2R reflect the total amount of the activated $\mathrm{T}$ lymphocytes in tumor-infiltrating lymphocytes of cancer tissues and metastatic organs, because a part of alpha-chain of sIL-2R is released into the bloodstream on the attachment of IL-2 to its specific sIL-2R membrane [7]. Interleukin-8 (IL-8) chemokine for neutrophils and lymphocyte has been demonstrated to be produced by bone marrow stromal cells [8]. Hypothesis suggested that IL-8 may be able to attract circulating malignant plasma cells precursors into an IL-6-rich bone marrow microenvironment [6]. Recently, high-dose therapy (HDT) with 
autologous stem cell transplantation (auto-SCT) has become the standard treatment for many patients with MM. Hence HDT and autologous stem cell rescue are considered to be a standard part of initial therapy for patients with MM $[9,10]$. Therefore, the patients accepted as potential transplant candidates are generally treated with dexamethasone-based programs rather than alkylating agents to avoid stem cell toxicity. Chemotherapy consisting of VAD (vincristine-adriamycin-dexamethasone) has commonly been used as the initial chemotherapeutic regimen in MM patients prior to HDT and auto-SCT $[10,11]$.

The objectives of the present study were to investigate circulating levels of mediators such as IL- $1 \beta$, sIL-2R, IL-6, IL- 8 , TNF- $\alpha$ as well as CRP before treatment and in different time periods after VAD treatment in patients with MM.

\section{MATERIALS AND METHODS}

This study involved a total of 14 newly diagnosed MM patients who were admitted to the Department of Hematology, Turgut Ozal Medical Center, Inonu University, Malatya, Turkey. They were 6 males $(42 \%)$ and 8 females $(58 \%)$. The mean age was $63.4 \pm 10.8$ (range $42-$ 71) years. The patients were in stage III of MM according to the Durie-Salmon classification. The study was carried out in compliance with the guidelines prescribed by the Institutional Health Committee at the Medical Faculty, Inonu University. All of the patients received VAD and bisphosphonates (pamidronate) treatment following diagnosis. Venous blood samples were taken before initiation of VAD therapy ( 0 day) and on the $3 \mathrm{rd}$, $7 \mathrm{th}, 10 \mathrm{th}$, and 28 th days after the first course of VAD. The VAD regimen consisted of vincristine $(0.4 \mathrm{mg} /$ day for 4 days IV), doxorubicin $\left(9 \mathrm{mg} / \mathrm{m}_{2} /\right.$ day for 4 days IV), and dexamethasone $(40 \mathrm{mg} /$ day per orally on days $1-4,9-12$, and $17-$ $20)$. The treatment cycles were repeated at 4 -week intervals. None of the patients or healthy controls was receiving any topical or systemic medication on admission. Following centrifugation of the blood samples at $2000 \mathrm{xg}$ for 10 minutes, sera were separated and kept at $-70^{\circ} \mathrm{C}$ until the analysis of cytokines.

\section{Cytokine analysis}

The analyses were performed using the Immulite chemiluminescent enzyme immunometric assay (Diagnostic Products, Los Angeles, Calif). The technique was based on a solid-phase (bead) two-site chemiluminescent enzyme immunometric assay. For each cytokine calibration, a master curve was constructed according to remarks of the National Institute for Biological Standards and manufacturer's instructions. TNF- $\alpha$ assay has a calibration range of up to $1000 \mathrm{pg} / \mathrm{mL}$. The assay is standardized in terms of the National Institute for Biological Standards and Control reference preparation number $87 / 650$. The detection limit of the assay was approximately $1.7 \mathrm{pg} / \mathrm{mL}$.
IL- $1 \beta$ assay has a calibration range up to $1000 \mathrm{pg} / \mathrm{mL}$. The assay is standardized in terms of the international standard for IL- $1 \beta, 86 / 680$. The detection limit of the assay was approximately $1.5 \mathrm{pg} / \mathrm{mL}$. sIL-2R assay has a calibration range up to $7200 \mathrm{U} / \mathrm{mL}$. The detection limit of the assay is approximately $10 \mathrm{U} / \mathrm{mL}$. IL- 6 assay has a calibration range up to $2000 \mathrm{pg} / \mathrm{mL}$. The assay is standardized in terms of the international standard for IL-6, 89/548. The detection limit of the assay was approximately $1 \mathrm{pg} / \mathrm{mL}$. IL- 8 assay has a calibration range up to $7500 \mathrm{pg} / \mathrm{mL}$. The assay is standardized in terms of the National Institute for Biological Standards and Controls reference preparation number $89 / 520$. The detection limit of the assay was approximately $2 \mathrm{pg} / \mathrm{mL}$. The coefficient of variance of intraassay and interassay was generally in the range of $4.3 \%$ $9.7 \%$.

\section{Statistical analysis}

Mean and standard deviations were calculated for each parameter. Variable normality analysis was performed. Variables in Table 1 showed normal distribution and comparison of means calculated by parametric unpaired $t$ test. Whereas parameters in Table 2 did not show normal distribution and repeated measures, ANOVA test was done. Pearson's linear correlation test was used for assessment of correlation between parameters. The minimum level of significance was defined at $P<.05$. All the above-mentioned analysis was performed using the Standard Package for Social Sciences (SPSS) version 9.0 for Windows (SPSS, Chicago, Ill).

\section{RESULTS}

The levels of cytokines and CRP were determined in newly diagnosed patients prior to and after VAD therapy. The results were expressed as means and standard deviation (SD). Summaries of the concentrations of the measured parameters prior to treatment are provided in Table 1. Pretreatment cytokine levels and CRP were found to be increased at diagnosis but significantly decreased 3, 7, and 10 days after VAD treatment (Table 2 ). The decrease in cytokine levels temporarily the occurred since the studied values started to increase on the 28th day after VAD therapy. Average levels of TNF- $\alpha$, IL- $1 \beta$, sIL-2R, IL6 , IL-8, and CRP differed according to the days after conducting VAD treatment. All cytokines except IL- $1 \beta$ were found to be positively correlated with CRP. Repeated measures ANOVA test for measured cytokines and CRP within and between different periods can be seen in Table 2 .

\section{DISCUSSION}

The significant point in this study is that serum levels of IL- $1 \beta$, sIL-2R, IL-6, IL- 8 , TNF- $\alpha$, and CRP in newly diagnosed stage III MM patients were significantly higher compared to normal controls and were significantly lower after chemotherapy. IL-6 is an important multifunctional 
TABLE 1. Comparison of serum levels of cytokines and CRP (mean \pm SD) in patients with MM.

\begin{tabular}{lccc}
\hline Parameters & Control & Patients & $P$ value \\
\hline TNF- $\alpha(\mathrm{pg} / \mathrm{mL})$ & $6.3 \pm 1.3$ & $18.6 \pm 3.7$ & $P<.0001$ \\
IL-1 $\beta(\mathrm{pg} / \mathrm{mL})$ & $4.3 \pm 0.2$ & $10.1 \pm 2.8$ & $P<.0001$ \\
sIL-2R $(\mathrm{U} / \mathrm{mL})$ & $597 \pm 80$ & $730 \pm 220$ & $P=.021$ \\
IL-6 $(\mathrm{pg} / \mathrm{mL})$ & $4.4 \pm 0.2$ & $11.4 \pm 3.2$ & $P<.0001$ \\
IL-8 $(\mathrm{pg} / \mathrm{mL})$ & $6.4 \pm 1.6$ & $23.9 \pm 8.3$ & $P<.0001$ \\
CRP $(\mathrm{mg} / \mathrm{dL})$ & $4.1 \pm 1.5$ & $49.9 \pm 19.4$ & $P<.0001$ \\
\hline
\end{tabular}

TABLE 2. Serum cytokines and CRP levels $($ mean \pm SD) in different periods after VAD treatment.

\begin{tabular}{lcccccc}
\hline Periods & TNF- $\alpha(\mathrm{pg} / \mathrm{mL})$ & IL- $\beta(\mathrm{pg} / \mathrm{mL})$ & sIL-2R $(\mathrm{U} / \mathrm{mL})$ & IL-6 $(\mathrm{pg} / \mathrm{mL})$ & IL-8 $(\mathrm{pg} / \mathrm{mL})$ & $\mathrm{CRP}(\mathrm{mg} / \mathrm{dL})$ \\
\hline Before treatment (I) & $18.6 \pm 3.7$ & $10.1 \pm 2.8$ & $730 \pm 220$ & $11.4 \pm 3.3$ & $23.9 \pm 8.3$ & $49.9 \pm 19.5$ \\
3 days after treatment (II) & $13.6 \pm 4.3$ & $6.5 \pm 1.1$ & $670 \pm 340$ & $7.1 \pm 1.4$ & $16.2 \pm 5.9$ & $25.8 \pm 8.3$ \\
7 days after treatment (III) & $11.9 \pm 3.9$ & $5.9 \pm 0.6$ & $540 \pm 125$ & $5.2 \pm 0.8$ & $13.5 \pm 4.2$ & $3.1 \pm 1.2$ \\
10 days after treatment (IV) & $8.1 \pm 1.1$ & $5.9 \pm 0.6$ & $436 \pm 28$ & $4.6 \pm 0.5$ & $5.2 \pm 0.7$ & $1.8 \pm 2.5$ \\
28 days after treatment (V) & $11.8 \pm 2.5$ & $8.8 \pm 2.7$ & $779 \pm 97$ & $7.5 \pm 1.2$ & $8.6 \pm 1.7$ & $23.9 \pm 10.9$ \\
Repeated measures & II-V & I-V, III-IV & I-II, I-V, & II-V, III-IV & None & II-V \\
ANOVA $P>.05$ & \multirow{2}{*}{ Rest } & Rest & Rest & Rest & All & Rest \\
Repeated measures & & & & & & \\
ANOVA $P<.05$ & & & & & &
\end{tabular}

proinflammatory cytokine involved in tumor growth and metastasis [12]. IL-6 is also the major growth and survival factor for MM and has been shown to protect MM cells from apoptosis induced by a variety of agents $[13,14]$. Serum and bone marrow IL-6 levels were found to be elevated in myeloma patients, and levels correlate with disease activity and disease status [6]. CRP serum levels reflect IL-6 in vivo and it may be regarded as a powerful prognostic factor in patients with MM $[5,15]$. TNF- $\alpha$ increases in all types of cellular and humoral immune response and shows a synergistic effect with IL-6 $[4,16]$. IL$1 \beta$ and TNF- $\alpha$ are also potent bone-resorbing cytokines that may contribute to the development of the osteolytic bone disease observed in patients with $\mathrm{MM}[2,4]$. Increased proinflammatory cytokines (IL- $1 \beta$, IL-6, TNF- $\alpha$ ) and CRP serum levels have been reported in different series of MM patients $[1,2,3,4,6,17]$. Similar to the previous findings from literature, our results showed that these proinflammatory cytokines were higher in the MM patients than control groups. Bisphosphonates seem to have an anti-inflammatory activity caused by the inhibition of the release of inflammatory mediators from activated macrophages, such as IL- 6 , IL- $1 \beta$, TNF- $\alpha$, and CRP $[18,19,20,21]$. Several studies have demonstrated bisphosphonates to be powerful inhibitors of bone resorption $[19,20,21]$. According to our results, the combination of $\mathrm{VAD} /$ bisphosphonate chemotherapy produced a significant reduction of the circulating serum levels of the cytokines.

There are sufficient data for serum IL-8 and sIL$2 \mathrm{R}$ levels of MM patients before and after treatment in the literature. IL- 8 plays a crucial role in inflammatory and tumor-associated angiogenesis, as well as in tumor progression $[8,22]$. Bone marrow stromal cells (BMSC) from myeloma patients also aberrantly secrete the chemokine IL- $8[8,23]$. It is possible that this aberrant production of IL- 8 by the BMSC of patients with myeloma does not have a direct effect on myeloma growth and survival per se but may instead function to promote angiogenesis $[8,23,24]$. Nevertheless, according to literature reviews although some mediators were studied in MM, sIL-2R, and IL- 8 in particular, have not yet been investigated in $\mathrm{MM}$ in relation to chemotherapy. High levels of sIL-2R have been measured in many inflammatory and malignant diseases $[6,7]$. Although the specific role of sIL-2R in the immune reponse has not yet been fully described, elevated serum sIL-2R levels are correlated with MM activity and MM stage $[25,26]$. Experimental data from a study conducted by Vacca et al report that serum and urinary values of sIL-2R were significantly increased in MM patients compared with normal controls [26]. Our results showed that circulating serum levels of the cytokines sIL-2R and IL- 8 were increased in MM patients compared to controls and were positively correlated with the other cytokines. Circulating serum levels of the cytokines sIL-2R and IL-8 decreased significantly following chemotherapy. Our study is the first one demonstrating reduction of circulating serum mediators, sIL-2R and IL-8 levels, after chemotherapy.

In summary, our data also supported involvement of several mediators in the pathogenesis of MM. Decreased circulating serum mediators of sIL-2R and IL-8 levels may also give a preliminary idea of the assessment of therapy response. Our results also suggest that after a time interval (10-28 days), cytokine levels reincrease. This could mean that cytokines suppression was temporary and one 
VAD therapy course of recommended dose and period is likely inadequate. Repeated VAD/bisphosphonate courses may provide better cytokines suppression. So, more efforts should be directed not only toward understanding the significance of the variability of these cytokines in patients with MM, but also their potential role in therapeutic approaches to MM. Further investigations are warranted in larger patient groups to support our present findings.

\section{REFERENCES}

[1] Hsu JH, Shi Y, Frost P, et al. Interleukin-6 activates phosphoinositol-3' kinase in multiple myeloma tumor cells by signaling through RAS-dependent and, separately, through p85-dependent pathways. Oncogene. 2004;23(19):3368-3375.

[2] Abildgaard N, Glerup H, Rungby J, et al. Biochemical markers of bone metabolism reflect osteoclastic and osteoblastic activity in multiple myeloma. Eur J Haematol. 2000;64(2):121-129.

[3] Lust JA, Donovan KA. The role of interleukin-1 beta in the pathogenesis of multiple myeloma. Hematol Oncol Clin North Am. 1999;13(6):1117-1125.

[4] Sati HI, Greaves M, Apperley JF, Russell RG, Croucher PI. Expression of interleukin-1beta and tumour necrosis factor-alpha in plasma cells from patients with multiple myeloma. $\mathrm{Br} J$ Haematol. 1999;104(2):350-357.

[5] Biro L, Domjan G, Falus A, et al. Cytokine regulation of the acute-phase protein levels in multiple myeloma. Eur J Clin Invest. 1998;28(8):679-686.

[6] Lauta VM. A review of the cytokine network in multiple myeloma: diagnostic, prognostic, and therapeutic implications. Cancer. 2003;97(10):24402452.

[7] Murakami S. Soluble interleukin-2 receptor in cancer. Front Biosci. 2004;9:3085-3090.

[8] Merico F, Bergui L, Gregoretti MG, et al. Cytokines involved in the progression of multiple myeloma. Clin Exp Immunol. 1993;92(1):27-31.

[9] Caldera H, Giralt S. Stem cell transplantation for multiple myeloma: current status and future directions. Curr Hematol Rep. 2004;3(4):249-256.

[10] Kumar S, Lacy MQ, Dispenzieri A, et al. High-dose therapy and autologous stem cell transplantation for multiple myeloma poorly responsive to initial therapy. Bone Marrow Transplant. 2004;34(2):161-167.

[11] Kyle RA. Management of patients with multiple myeloma: emphasizing the role of high-dose therapy. Clin Lymphoma. 2001;2(1):21-28.

[12] Michalaki V, Syrigos K, Charles P, Waxman J. Serum levels of IL-6 and TNF-alpha correlate with clinicopathological features and patient survival in patients with prostate cancer. Br J Cancer. 2004;90(12):23122316.
[13] Wang YD, De Vos J, Jourdan M, et al. Cooperation between heparin-binding EGF-like growth factor and interleukin-6 in promoting the growth of human myeloma cells. Oncogene. 2002;21(16):25842592.

[14] Klein B, Tarte K, Jourdan M, et al. Survival and proliferation factors of normal and malignant plasma cells. Int J Hematol. 2003;78(2):106-113.

[15] Alexandrakis MG, Passam FH, Ganotakis ES, et al. The clinical and prognostic significance of erythrocyte sedimentation rate (ESR), serum interleukin6 (IL-6) and acute phase protein levels in multiple myeloma. Clin Lab Haematol. 2003;25(1):41-46.

[16] Silvestris F, Cafforio P, Calvani N, Dammacco F. Impaired osteoblastogenesis in myeloma bone disease: role of upregulated apoptosis by cytokines and malignant plasma cells. Br J Haematol. 2004;126(4):475-486.

[17] Rosinol L, Cibeira MT, Segarra M, et al. Response to thalidomide in multiple myeloma: impact of angiogenic factors. Cytokine. 2004;26(4):145-148.

[18] Terpos E, de la Fuente J, Szydlo R, et al. Tartrateresistant acid phosphatase isoform 5b: a novel serum marker for monitoring bone disease in multiple myeloma. Int J Cancer. 2003;106(3):455-457.

[19] Berenson JR. New advances in the biology and treatment of myeloma bone disease. Semin Hematol. 2001;38(suppl 3):15-20.

[20] Terpos E, Viniou N, de la Fuente J, et al. Pamidronate is superior to ibandronate in decreasing bone resorption, interleukin-6 and beta 2-microglobulin in multiple myeloma. Eur J Haematol. 2003;70(1):3442.

[21] Santini D, Fratto ME, Vincenzi B, La Cesa A, Dianzani C, Tonini G. Bisphosphonate effects in cancer and inflammatory diseases: in vitro and in vivo modulation of cytokine activities. BioDrugs. 2004;18(4):269-278.

[22] Strieter RM, Polverini PJ, Arenberg DA, et al. Role of $\mathrm{C}-\mathrm{X}-\mathrm{C}$ chemokines as regulators of angiogenesis in lung cancer. J Leukoc Biol. 1995;57(5):752-762.

[23] Rossi D, Zlotnik A. The biology of chemokines and their receptors. Annu Rev Immunol. 2000;18:217242.

[24] Sparmann A, Bar-Sagi D. Ras-induced interleukin-8 expression plays a critical role in tumor growth and angiogenesis. Cancer Cell. 2004;6(5):447-458.

[25] Filella X, Blade J, Guillermo AL, Molina R, Rozman C, Ballesta AM. Cytokines (IL-6, TNF-alpha, ILlalpha) and soluble interleukin-2 receptor as serum tumor markers in multiple myeloma. Cancer Detect Prev. 1996;20(1):52-56.

[26] Vacca A, Di Stefano R, Frassanito A, Iodice G, Dammacco F. A disturbance of the IL-2/IL-2 receptor system parallels the activity of multiple myeloma. Clin Exp Immunol. 1991;84(3):429-434. 


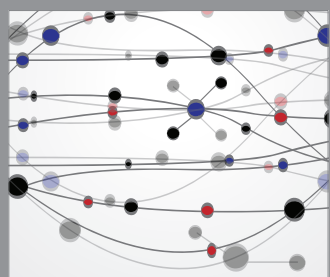

The Scientific World Journal
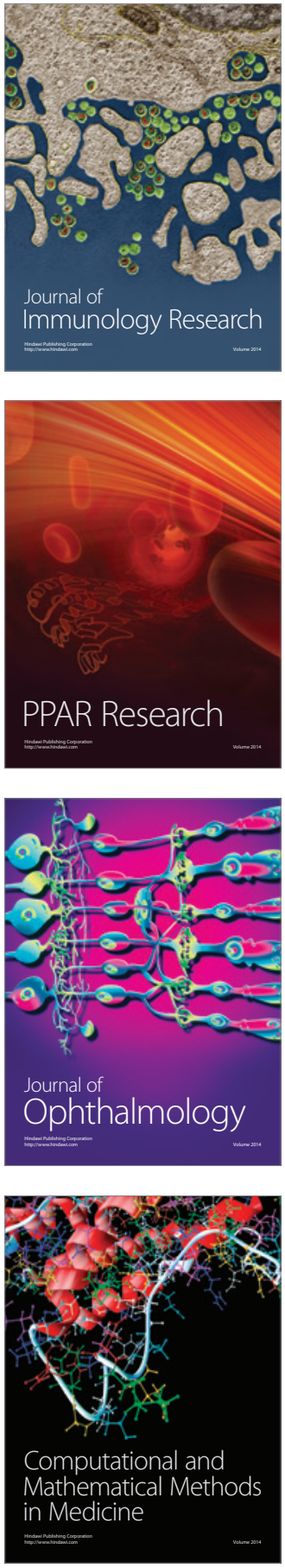

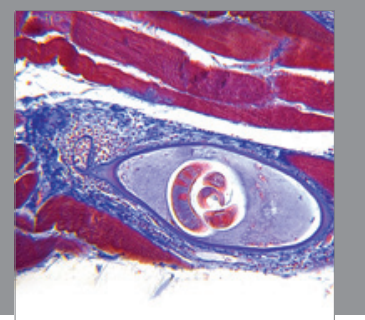

Gastroenterology

Research and Practice
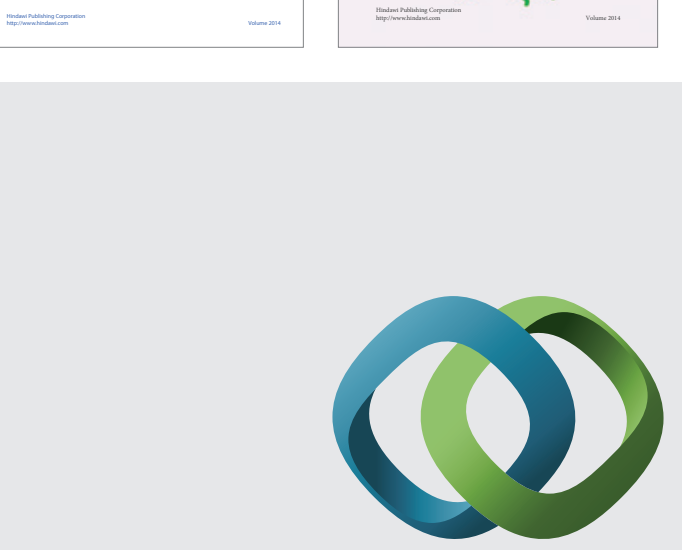

\section{Hindawi}

Submit your manuscripts at

http://www.hindawi.com
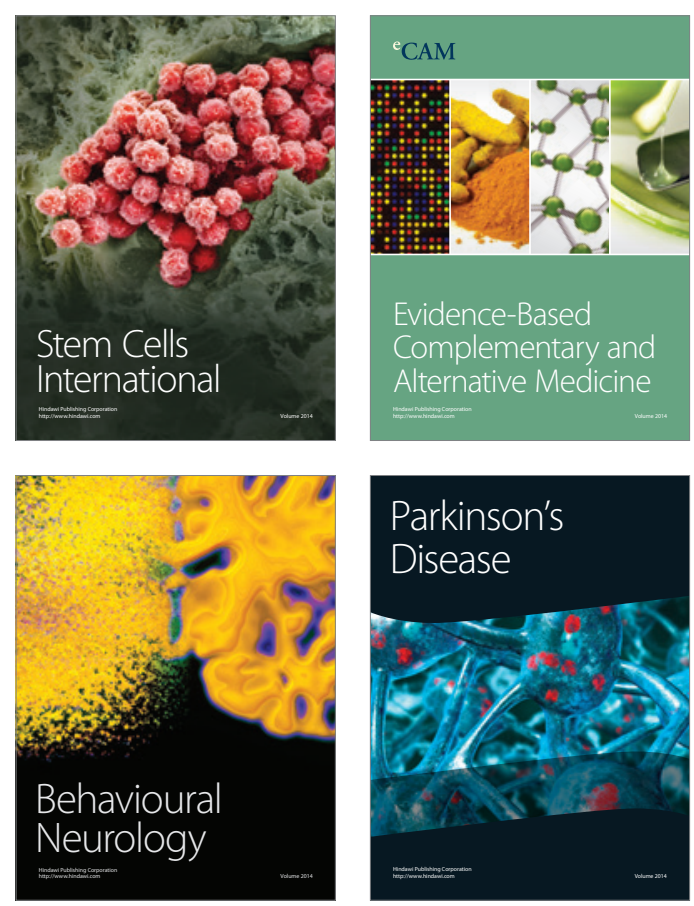

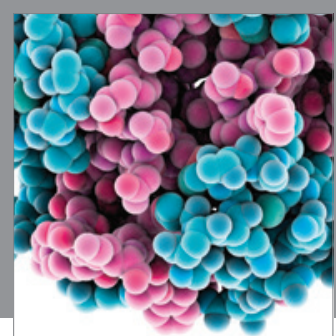

Journal of
Diabetes Research

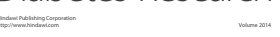

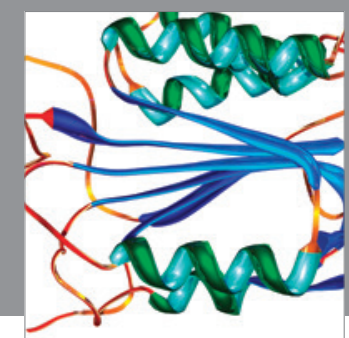

Disease Markers
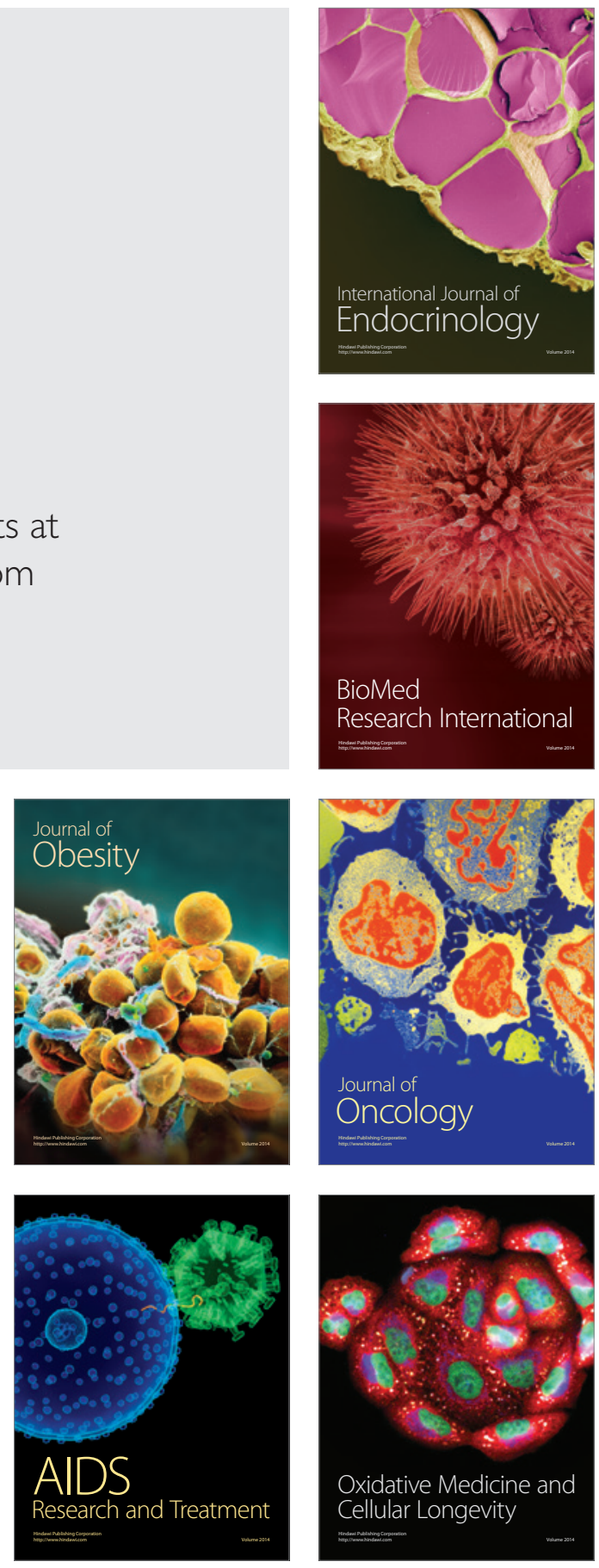